\title{
The broad-band X-ray spectrum of Mrk 3
}

\author{
R. G. Griffiths ${ }^{1}$, R. S. Warwick ${ }^{1}$, I. Georgantopoulos ${ }^{1,2}$, C. Done ${ }^{3}$ and \\ D. A. Smith ${ }^{1}$ \\ ${ }^{1}$ Department of Physics and Astronomy, University of Leicester, Leicester, LE1 7RH \\ ${ }^{2}$ Astronomical Institute, National Observatory of Athens, Lofos Koufou, Palaia Penteli, Athens, GR - 15236, Greece \\ ${ }^{3}$ Department of Physics, University of Durham, South Road, Durham DH1 3LE
}

4 April 2018

\begin{abstract}
We have used non-simultaneous Ginga, ASCA and ROSAT observations to investigate the complex X-ray spectrum of the Seyfert 2 galaxy Mrk 3. We find that the composite spectrum can be well described in terms of a heavily cut-off hard X-ray continuum, iron $K_{\alpha}$ emission and a soft X-ray excess, with spectral variability confined to changes in the continuum normalisation and the flux in the iron line. Previous studies have suggested that the power-law continuum in Mrk 3 is unusually hard. We obtain a canonical value for the energy index of the continuum (i.e. $\alpha \approx 0.7$ ) when a warm absorber (responsible for an absorption edge observed near $8 \mathrm{keV}$ ) is included in the spectral model. Alternatively, the inclusion of a reflection component yields a comparable power-law index. The soft-excess flux cannot be modelled solely in terms of pure electron scattering of the underlying power-law continuum. However, a better fit to the spectral data is obtained if we include the effects of both emission and absorption in a partially photoionized scattering medium. In particular the spectral feature prominent at $\sim 0.9 \mathrm{keV}$ could represent $\mathrm{O}$ VIII recombination radiation produced in a hot photoionized medium. We discuss our results in the context of other recent studies of the soft X-ray spectra of Seyfert 2 galaxies.
\end{abstract}

Key words: galaxies:active - galaxies:Seyfert - X-rays:galaxies.

\section{INTRODUCTION}

Seyfert galaxies have been observed extensively in the high - energy band to reveal a host of interesting properties. Seyfert 1 galaxies are typically much brighter X-ray sources than Seyfert 2's, a result which is explained by current unification models as primarily due to the effects of obscuration and orientation (e.g. Antonucci 1993). Both types of object are presumed to contain an active galactic nucleus (AGN) comprising a supermassive blackhole, an accretion disk, a broad-line region and (in many cases) a thick molecular torus. According to the unified scheme, we have a relatively clear view of the AGN in a Seyfert 1 galaxy, whereas in Seyfert 2 galaxies the torus is observed at sufficiently high inclination so as to introduce significant obscuration into the direct line-of-sight to the central source. Recent X-ray spectral observations provide clear evidence for highly ionized absorption systems (i.e. warm absorbers) in many Seyfert 1 galaxies (Reynolds 1997; George et al. 1998). It appears that this ionized material extends sufficiently far from the nucleus along the axial direction, so as to be visible above the torus even when the system is viewed close to edge-on. In Seyfert 2's this medium can scatter nuclear flux into our line-of-sight, even when our direct view of the nucleus is en- tirely blocked by the torus (e.g. NGC 1068, Antonucci \& Miller 1985).

As a result of missions such as Ginga, ROSAT and $A S C A$ and, more recently, RXTE and SAX, Seyfert 1's and Seyfert 2's have been shown to exhibit a rich phenomenology in terms of their X-ray spectra. Recent interest has centred on (i) the temporal and spectral properties of the hard $\mathrm{X}$-ray continuum produced close to the supermassive blackhole; (ii) the presence of spectral components resulting from the Compton reflection of continuum flux by optically thick matter in the proximity of the central source; (iii) the detailed study of the iron $\mathrm{K}_{\alpha}$ line (in particular the presence of broad-line profiles commensurate with predicted gravitational redshifts and Doppler broadening effects) ; (iv) the presence of highly ionized material whether viewed directly against the continuum source as a 'warm-absorber' or, indirectly, via scattered nuclear emission in those sources where the direct soft X-ray continuum is strongly cut-off due to line-of-sight absorption.

In the present paper we focus on the X-ray properties of the Seyfert 2 galaxy, Mrk 3. Our approach is to use nonsimultaneous Ginga, ASCA and ROSAT observations to secure broad (0.1-30 keV) spectral coverage. Although Mrk 3 
has previously been studied in some detail on the basis of the individual datasets (Awaki et al. 1990; Awaki et al. 1991; Iwasawa et al. 1994; Turner, Urry \& Mushotzky 1993; Smith \& Done 1996; Turner et al. 1997a,b), the combination of the data from the three satellites provides clear benefits in terms of spectral modelling. For example, the ASCA instruments, despite their superior spectral resolution, do not have the spectral bandwidth to constrain the slope of the underlying hard X-ray continuum in a source, such as Mrk 3, where this continuum is strongly cut-off in the soft X-ray regime. However, once the continuum form is reasonably specified by the inclusion of Ginga data, then the ASCA observations provide tight constraints on sharp emission features. Similarly the inclusion of ROSAT data extends the spectral coverage below $\sim 0.6 \mathrm{keV}$ to give additional leverage when modelling the soft X-ray spectral form. The consideration of the composite spectrum also allows the possibility of spectral variability to be investigated in a consistent manner.

One particular point of interest in the case of Mrk 3 is that it appears to have a rather hard continuum form. The Ginga observations (Awaki et al. 1990; Awaki et al. 1991) reveal a power-law index $\alpha \approx 0.5$, which is somewhat harder than is typical of Seyfert 1 galaxies (e.g. Nandra \& Pounds 1994) and fairly extreme amongst the sample of $\sim 16$ Seyfert 2's studied by Ginga (Smith \& Done 1996). In principle the observation of anomalously flat power-law slopes, even in a subset of Seyfert 2 galaxies (NGC5252 provides a further example; Cappi et al. 1996) could be interpreted as contradicting unification models. Flat spectrum populations are also of general interest in terms of their potential contribution to the cosmic X-ray background (XRB).

This paper is organised as follows. Brief details of the observations and data reduction methods are presented in section 2 . In the next section we describe the approach we have taken in modelling the various spectral datasets, including the use of the photoionization code XSTAR (Kallman \& Krolik 1997) to calculate the emergent spectrum from a partially photoionized scattering medium. In section 4 we discuss the implications of our results in the context of other, recently published studies of the X-ray spectral properties of Seyfert 2 galaxies. Finally we summarise our conclusions in section 5 .

\section{OBSERVATIONS AND DATA REDUCTION}

\subsection{The Ginga observations}

Mrk 3 was observed with the Large Area Counter (LAC, Turner et al. 1989) on board the Ginga satellite (Makino et al. 1987) during the period September 27-28, 1989. The methods employed in the data selection, in the background subtraction and in correcting for pointing offsets were the same as those described in Smith \& Done (1996). X-ray pulse-height spectra were extracted from both the "top-" and "mid-layers" of the LAC; these counters provide spectral coverage across nominal $2-37 \mathrm{keV}$ and $6-37 \mathrm{keV}$ energy bands respectively. The effective integration time was $24 \mathrm{ks}$ and the average count rates were 4.6 count $\mathrm{s}^{-1}$ and 2.4 count $\mathrm{s}^{-1}$ in the top and mid-layer counters respectively. In the spectral analysis, data below $4.5 \mathrm{keV}$ were ignored because of the presence of anomalous line features at $\sim 3-4$
$\mathrm{keV}$ (possibly identified with argon $\mathrm{K}$ and xenon $\mathrm{L}$ lines in the LAC background; see Smith \& Done 1996) and high energy channels above $27 \mathrm{keV}$ were excluded due to their susceptibility to background subtraction errors. A one percent systematic error was added to each pulse-height channel to account for uncertainties in the detector response.

\subsection{The ASCA observations}

ASCA observed Mrk 3 during the period April 21-22, 1993. There are four instruments on-board ASCA, two Solid-State Imaging Spectrometers (SIS-0 and SIS-1) and two Gas Imaging Spectrometers (GIS-2 and GIS-3) (Tanaka, Inoue \& Holt 1994). The SIS data were taken in 4-CCD FAINT and BRIGHT modes. We combined FAINT mode data (after conversion to BRIGHT mode), and true BRIGHT mode data. This procedure does not exploit the maximum energy resolution of the SIS, but it does increase the signal-tonoise ratio. (We obtained $\sim 40 \%$ more counts by using the combined true and converted BRIGHT mode data rather than the FAINT mode data alone). The events lists have been screened using the standard ASCA analysis procedure. Only events of grade $0,2,3$ and 4 have been used and data taken during intervals when the Earth elevation angle was less than $5^{\circ}$ and when the magnetic cut-off rigidity was less than $6 \mathrm{GeV} / \mathrm{c}$ were rejected. For the SIS data, "hot" and "flickering" pixels and telemetry saturated frames were also removed. In addition, counts were rejected when the bright Earth angle was less than $20^{\circ}$. This procedure resulted in 30 (42) ks of good SIS (GIS) data for Mrk 3.

Spectral data sets were obtained from each telescope by integrating events within a circular region (of radius 34 arcmin) centred on Mrk 3 and the background was estimated from source-free regions in the same observation. The average background-subtracted count rates for Mrk 3 were 0.05 count $\mathrm{s}^{-1}$ and 0.04 count $\mathrm{s}^{-1}$ in the SIS and GIS systems respectively. In the subsequent spectral analysis we have ignored all SIS (GIS) data below 0.6 (0.8) keV (since the uncertainty in the calibration is highest in these low energy channnels). The pulse-height spectra were binned so as to give at least 20 counts per spectral channel and, in the spectral analysis, the data from all four detectors were fitted simultaneously.

\subsection{The ROSAT/PSPC observations}

Mrk 3 was observed with the X-ray Telescope (XRT) and Position Sensitive Proportional Counter (PSPC) combination on board ROSAT during the period March 8-10, 1993. Data were rejected when the Master Veto rate exceeded a threshold of 170 count $\mathrm{s}^{-1}$, which resulted in a total exposure time of $\sim 15 \mathrm{ks}$. The background region was taken from an annulus with an inner radius of $9^{\prime}$ and outer radius of $15^{\prime}$, centred on Mrk 3, with all the confusing sources masked out. The observed $0.1-2.4 \mathrm{keV}$ PSPC count rate was 0.06 count $\mathrm{s}^{-1}$.

\section{SPECTRAL ANALYSIS}

In this section we first consider the Ginga spectra and $A S C A$ spectra separately so as to allow direct comparison with ear- 
lier studies. We then go on to apply more detailed spectral models to the composite Ginga, ASCA and ROSAT spectra. The spectral analysis was conducted using XSPEC version 10.0 .

We note that there is a faint X-ray source detected $\sim 1.7^{\prime}$ West of Mrk 3 in both the ROSAT PSPC (Turner et al. 1993) and HRI (Morse et al. 1995) images. The source flux is only $\sim 4.4 \%$ of the flux from Mrk 3 in the $0.1-2.4$ $\mathrm{keV}$ PSPC band. The presence of this faint source within the Ginga LAC field of view and in the ASCA "on-source" region is very unlikely to produce a significant effect in terms of our analysis of the Mrk 3 spectrum. (Furthermore the centroid of the source lies in the interchip gap for both the SIS0 and SIS1 detectors). Iwasawa et al. (1994) and Turner et al. (1997a) also report the detection of a BL Lac object, 10.6' NW of Mrk 3 in the ASCA data; this source also lies within the Ginga field of view. Based on the ASCA data we estimated that the count rate from the BL Lac object is roughly $2 \%$ of that of Mrk 3 in the $4-10 \mathrm{keV}$ band and hence the impact of this confusing source on the Ginga measurements will be small.

\subsection{The Ginga spectra}

The first model used to fit the Ginga LAC spectra comprised a single hard power-law continuum, with energy index $\alpha$ and normalisation $A_{\text {Ginga }}$, which is heavily cut-off at low energy due to absorption by cold solar abundance gas of column density, $N_{H}$. (Throughout this paper we have used the element abundances and the absorption cross-sections adopted by Morrison \& McCammon 1983). An iron $\mathrm{K}_{\alpha}$ emission line of intensity $A_{K \alpha}$ at an energy $E_{K \alpha}$ was also included with a width fixed at $\sigma_{K \alpha}=0.1 \mathrm{keV}$ (we use this value throughout, see section 4). This model (Model 1) provides a good match to the Ginga data for Mrk 3; Table 1 lists the best-fit parameters and associated errors (the errors quoted throughout this paper are 90 per cent confidence limits for one interesting parameter following the prescription of Lampton, Margon \& Bowyer 1976).

The parameter values derived on the basis of Model 1 agree reasonably well with earlier analyses of the Ginga (top-layer only) spectra (Awaki et al. 1990; Awaki et al. 1991). Specifically we confirm, on the basis of the Ginga data alone, the rather hard continuum spectrum (i.e. $\alpha=$ $0.37_{-0.14}^{+0.15}$ ). However, one apparent discrepancy is that Smith \& Done (1996) obtained $\alpha=-0.15_{-0.19}^{+0.13}$ for Mrk 3, which is a very much flatter continuum slope than quoted here. The origin of this problem can be traced to the fact that Smith \& Done restricted their analysis to the $2-18 \mathrm{keV}$ energy range whereas we use the $4.5-27 \mathrm{keV}$ band. If we employ Model 1 to fit the Ginga 2-18 keV top-layer data for Mrk 3, then we also obtain a peculiar spectral index. This emphasises the value of the high energy data, particularly that from the LAC mid-layer. (The mid-layer has a larger effective area than the top-layer above $\sim 10 \mathrm{keV}$ and therefore provides useful additional constraints on the slope of the hard X-ray continuum).

The measured Ginga count rate spectra, the best-fitting spectral model and the spectral fitting residuals are shown in Fig. 1. The residuals suggest the possible presence of an additional absorption edge at $\sim 8 \mathrm{keV}$. When such a feature is included a reduction in $\chi^{2}$ of $\sim 14$ is obtained with two additional parameters, the edge energy $E_{\text {edge }}$ and optical depth $\tau_{\text {edge }}$ (Model 2, Table 1), which in terms of the F-test is significant at the $>99 \%$ level. One effect of this additional iron absorption is to steepen the continuum by $\Delta \alpha \approx 0.3$. The measured edge energy, $E_{\text {edge }}=8.1_{-0.5}^{+0.4} \mathrm{keV}$, is consistent with the $\mathrm{K}$-edge of iron in an ionization state in the range Fe XVIII-XXIII, implying the presence of highly ionized gas in the line-of-sight to the hard X-ray source in addition to the large column density of relatively cold gas. The possibility of additional line-of-sight absorption by photoionized gas is discussed further in section 3.3.

\subsection{The ASCA spectra}

Mrk 3 exhibits excess soft X-ray emission over and above that expected given the heavy absorption evident in the Ginga passband. To account for this soft X-ray emission the spectral form used to fit the ASCA SIS and GIS data was basically that of Model 1 (with free parameters $\alpha, A_{A S C A}$, $\left.N_{H}, A_{K \alpha}, E_{K \alpha}\right)$ plus a second power-law with the same spectral slope as the intrinsic hard continuum but with a different normalisation $\left(A_{\text {soft }}\right)$. Hereafter we will refer to this as the soft power-law component. In physical terms this soft power-law could be flux leakage from a partially covered source with an uncovered fraction $f=A_{\text {soft }} / A_{A S C A}$. Alternatively $f$ could represent the fraction of the continuum flux which is directed into our line of sight by an extended electron scattering region (i.e. $f \equiv f_{\text {scat }}$ ). We will, in fact, pursue this latter interpretation in the current paper. We assumed that the soft power-law component is absorbed only by the Galactic foreground HI column density of $N_{\text {gal }}=8.7 \times 10^{20} \mathrm{~cm}^{-2}$ (Elvis, Lockman \& Wilkes 1989).

The above spectral prescription provides only a modestly successful fit to the ASCA data (Model 3, Table 2). Significant residuals are evident at a variety of energies in the ASCA detectors. Previously Iwasawa et al. (1994) have reported the presence of line-like features at $\sim 0.75 \mathrm{keV}$, $0.9 \mathrm{keV}$ and possibly $\sim 7.0 \mathrm{keV}$ in the SIS spectra of Mrk 3 (in addition to the $6.4 \mathrm{keV}$ line included in Model 3). Similarly Turner et al. (1997a) found that the addition of narrow lines at $1.85,2.45,6.4$ and $6.96 \mathrm{keV}$ (corresponding to SiXIII, SXV, FeI-XVI and FeXXVI respectively) to their best-fitting double power-law continuum model, significantly improved the fit to the ASCA data. The significance of any residual features is, of course, a strong function of the continuum model fitted (see Turner et al. 1997a).

We obtain an improved fit if we include a solarabundance Raymond-Smith component (temperature $k T_{\text {Ray }}$ and normalisation $A_{R a y}$ ), in addition to the soft power-law (Model 4, Table 2). One aspect to note is that Model 4 gives a much flatter power-law slope than Model 3. The difference appears to arise from the fact that in Model 3 the intrinsic and soft power-laws are constrained to have the same spectral index and the steepness of the soft excess tends to pull the overall continuum form to a steeper slope, whereas when the Raymond-Smith component is included this linkage is much weaker. However, the soft X-ray power-law component is still essential since fits of Model 4 with $A_{\text {soft }}$ set to zero give a worse result than even Model $3\left(\chi^{2} /\right.$ d.o.f. $=$ $443 / 299$ compared to $\chi^{2} /$ d.o.f. $\left.=390 / 300\right)$.

In the next section we focus our attention on the possibility of fitting some of the line-like features with a model 
which includes the effects of photoionization on the putative scattering medium (and hence removes the requirement for a Raymond-Smith component). For completeness we include the results of fitting such a model to the ASCA-only data as Model 5 in Table 3 (details of this model are explained in the next section). The fact that this photoionization model gives a rather similar $\chi^{2}$ to Model 4 suggests that it is difficult to distinguish between the two descriptions at the limits of the sensitivity and spectral resolution afforded by ASCA.

\subsection{The composite Ginga, ASCA and ROSAT spectra}

Since the Ginga, ASCA and ROSAT observations were not recorded contemporaneously (the observations were, in fact, made over a period of $\sim 4$ years) our analysis is based on the assumption that the spectral form of Mrk 3 is largely invariant. Thus in the spectral analysis we allow only the normalisation of the intrinsic power-law continuum and the flux of the iron $\mathrm{K}_{\alpha}$ line to vary between the Ginga and ASCA datasets; the spectral parameters used to model the ROSAT PSPC spectrum were identical to those applied to the ASCA data. We will check this assumption later when considering the quality of the model fit to the composite spectra.

Table 3 gives the results of fitting Models 3 and 4 to the composite spectra for Mrk 3. The results generally mirror those obtained from the ASCA spectra alone, although as noted earlier the Ginga data prefer a rather harder power law and this influence is evident from a comparison of the results in Table 3 with those in Table 2. By way of illustration Fig. 2 shows the unfolded spectral data based on Model 3 and the corresponding count rate residuals to the composite spectrum. Interestingly the residual feature near $0.9 \mathrm{keV}$ identified by Isawawa et al. (1994) appears to be present both in the ASCA and ROSAT data. This is also consistent with the report by Turner et al. (1993) of the presence a blend of soft X-ray lines below $\sim 1 \mathrm{keV}$ in the ROSAT PSPC spectrum of Mrk 3. Other distinctive line features are less obvious. For example, both Iwasawa et al. (1994) and Turner et al. (1997a) report the presence of a line near $7.0 \mathrm{keV}$ which could be $\mathrm{K}_{\alpha}$ emission from hydrogen-like iron. We have investigated whether a narrow line is present at $6.87 \mathrm{keV}$ (i.e. the redshifted energy of the Fe XXVI line) but find that this line is at best $\sim 20$ times weaker than the $6.4 \mathrm{keV}$ line and that its inclusion gives no significant improvement in the spectral fitting. Iwasawa et al. (1994) note that in their analysis this line is only marginally detected. We conclude that this is a case where the continuum model has a crucial bearing on the inferred significance of a line feature.

The inclusion of the solar-abundance Raymond-Smith component in addition to the soft power-law continuum (Model 4) provides a dramatic improvement in the fit to the soft X-ray spectrum of Mrk 3. Figure 3(a) illustrates the form of this spectral model in the $0.3-3 \mathrm{keV}$ band. With the ASCA spectral resolution, the line emission from the $\sim 0.7 \mathrm{keV}$ thermal component gives rise to a broad spectral enhancement in the range $0.7-1.0 \mathrm{keV}$ in rough accord with that observed. As a trial we reduced the elemental abundance in the Raymond-Smith model to 0.2 solar and refit the spectra. The effect was to increase the contribution of the thermal continuum to the soft X-ray spectrum, particu- larly below $0.6 \mathrm{keV}$. As a result, the slope of the power-law continuum flattens somewhat from $\alpha \approx 0.45$ to $\alpha \approx 0.37$ giving a slightly improved fit $\left(\Delta \chi^{2} \sim 7\right)$.

As discussed earlier we can interpret the soft power-law component in Models 3 and 4 as the fraction of the intrinsic hard X-ray continuum which is electron scattered into our line of sight. Unless the scattering region is completely photoionized by the incident nuclear flux, we would expect some absorption features to be imprinted on the scattered flux and also some line emission to emanate from the photoionized medium. Thus we now focus our attention on the possibility of extending Model 3 to include both absorption features in the scattered continuum and intrinsic emission from the scattering region.

We have used the photoionization code XSTAR (Kallman \& Krolik 1997) which models the emission and absorption of a cloud of photoionized gas. This photoionization code calculates the emission and absorption spectra produced by a gas cloud in the vicinity of a point source of continuum radiation. The assumed spectrum of the central ionizing source is the same one used by Krolik \& Kriss (1995) and is typical of Seyfert 1 galaxies and low-luminosity quasars. The state of the gas cloud is described by the ionization parameter $\xi=\mathrm{L} / n \mathrm{r}^{2}$ where $\mathrm{L}$ is the source luminosity in the 0.0136 - $13.6 \mathrm{keV}$ bandpass in erg $\mathrm{s}^{-1}, n$ is number of hydrogen atoms/ions in the gas per $\mathrm{cm}^{3}$ and $\mathrm{r}$ is the distance of the inner edge of the cloud to the central source in $\mathrm{cm}$. In our XSTAR models we have used a density of $n=10^{6} \mathrm{~cm}^{-3}$ and a gas temperature of $10^{6} \mathrm{~K}$ (but see section 4.2 ). The element abundances used are the default values in XSTAR. A range of ionization parameters $(\log \xi)$ and column densities $\left(N_{H_{\text {scat }}}\right)$ were used, from which a two-dimensional grid of spectral models was produced in the form of an "atable" and "mtable". The atable represents the intrinsic emission of the photoionized plasma, and the mtable specifies the fraction of the scattered power-law which emerges, after allowing for absorption, from the cloud. In both cases the tabulated spectral information encompassed an observational bandwidth from $0.1 \mathrm{keV}$ up to $40 \mathrm{keV}$. In the spectral fitting XSPEC interpolates between the grid points to obtain the best-fitting spectral parameters.

We assume that the absorption suffered by the scattered continuum is that arising in a slab of gas directly illuminated by the nuclear source in Mrk 3. The intrinsic emission from the scattering region is that emerging in the forward direction from this same slab. Clearly this approximation ignores a number of (unknown) geometrical factors relating to the spatial distribution of the scattering medium and our viewing orientation, but is adequate for the present purpose. Thus one set of spectral parameters apply to both the mtable and atable components. The three free parameters associated with the photoionized gas are, $\log \xi, N_{H_{\text {scat }}}$ and $A_{e m}$, the latter representing the normalisation of the emission spectrum风. In our modelling we have chosen not to directly couple the scattered fraction of continuum flux (i.e. $A_{\text {soft }}$ divided by either $A_{A S C A}$ or $A_{\text {Ginga }}$ ) with $A_{\text {em }}$ (see section 4.2).

The results of fitting this photoionization model are

\footnotetext{
* See the XSTAR manual for the definition of this normalisation parameter (Kallman \& Krolik 1997)
} 
given as Model 5 in Table 3 (and also in Table 2). This model gives a slightly better fit to the Mrk 3 spectrum than is the case for pure electron scattering plus a Raymond-Smith component (Model 4, Table 3). The form of the spectrum in $0.3-3 \mathrm{keV}$ band is shown in Figure $3(\mathrm{~b})$. The best-fitting model has $\log \xi \approx 2.8$ and reproduces the observational feature near $0.9 \mathrm{keV}$ as recombination directly into the K-shell of a completely stripped oxygen atom, a process which has a threshold energy of $0.87 \mathrm{keV}$. In Fig. 3(b) this O VIII recombination continuum feature is significantly broadened by the thermal energies of the free electrons in the $10^{6} \mathrm{~K}$ photoionized medium.

The derived slope of the intrinsic power-law continuum in Models 4 and 5 remains relatively flat $(\alpha \approx 0.45$ and $\approx 0.66$ respectively). One possible explanation of the apparent hardness of the underlying continuum in Mrk 3 is that Compton reflection makes a significant contribution to the observed flux above $\sim 6 \mathrm{keV}$ as appears often to be the case for Seyfert 1 galaxies (Pounds et al. 1990). We have investigated this possibility by including a Compton reflection component in the our spectral analysis; specifically we employ the XSPEC "pexrav" model. We assume that the reflection component is subject to absorption in the cold gas column density represented by $N_{H}$. In pexrav the parameter $R$ represents the relative strength of the reflected signal, with a value of unity corresponding to reflection from a semi-infinite flat disk of cold material which is irradiated by an isotropic power-law continuum source. Here, for simplicity, we fix the inclination angle of the disk at $\mathrm{i}=60^{\circ}$ and assume the same value of $R$ applies to the Ginga and ASCA observations. The results of including reflection in the photoionized scattering model are listed in Table 3 as Model 6 . The best-fitting version of Model 6 requires $R \sim 0.8$, (in the F-test the reduction in $\chi^{2}$ of $\sim 9$ is significant at the $>99$ $\%$ level for one additional parameter). Alternatively, when the relative strength of the reflected signal is frozen at $\mathrm{R}=$ 1 and the inclination angle of the disk allowed to vary, we obtain a best-fit value of $\mathrm{i}=71_{-16}^{+13} \mathrm{deg}$. The inclusion of reflection in the spectral model leads to a value for the spectral index of the hard continuum in Mrk 3 of $\alpha \approx 0.8$, close to the canonical value of 0.9 measured similarly in Seyfert 1 galaxies (Nandra \& Pounds 1994; Reynolds 1997; George et al. 1998).

In our earlier discussion of the Ginga spectra of Mrk 3 we noted the evidence for additional line-of-sight absorption (over and above that represented by $N_{H}$ ) in the form of an edge feature near $\sim 8 \mathrm{keV}$. If attributable to iron-K edge absorption, the implied ionization state of the absorbing gas is FeXVIII - FeXXIII. This is rather close to the state of the iron in the photoionized scattering region and suggests a possible model in which the scattering zone extends not only directly above the nuclear source (i.e. along the axial direction of the nuclear region) but also sufficiently close to the plane of the system to intercept our line of sight (the presumption being that we view the nucleus at fairly high inclination in the case of this "classical" Seyfert 2 galaxy). This "warm absorbing" medium would presumably lie inside the colder medium responsible for the bulk of the soft Xray cut-off, a situation which is quite plausible if the cold gas distribution is associated with a molecular torus in Mrk 3. We have investigated this twin absorbing-zone model by including a warm column density $N_{H_{\text {warm }}}$ in addition to the cold column $N_{H}$ in a revised version of Model 5. Again for simplicity we use the same mtable as described earlier and tie the ionization parameter of the warm absorber to that of the scattering medium. The results of this analysis are given in Table 3 as Model 7. We note that the fit is an improvement on both Models 5 and 6 . The inferred spectral index of the hard continuum in this "no-reflection" scenario is $\alpha \approx 0.7$; a value which was considered typical of the underlying hard continuum in Seyfert 1 galaxies prior to the realisation of the importance of the Compton reflection process (e.g. Turner $\&$ Pounds 1989). Finally we note that the requirement for a warm-absorber is not diminished if we add a reflection component to Model 7; however, we have not pursued this model further given its complexity in relation to the quality of the available data.

\section{DISCUSSION}

\subsection{Characteristics of the hard X-ray spectrum of Mrk 3}

Gross spectral variability in Mrk 3 might, in principle, be revealed as a mismatch between the Ginga, ASCA and ROSAT spectral datasets. In fact we obtain a rather good fit to the composite spectrum without the need for any crossinstrument scaling factors (this also demonstrates that the instrument calibrations are not too far out of line). We are thus able to verify our initial assumption that the spectral form of Mrk 3, within the limitations of the available observations, is largely invariant. Specifically the soft X-ray spectrum of Mrk 3 (below $\sim 3 \mathrm{keV}$ ) shows no significant change over the six week period separating the ROSAT and ASCA observations and similarly the form of the spectral cut-off due to photoelectric absorption of the hard continuum flux remained relatively constant over the 3.5 year period between the Ginga and $A S C A$ observations. In combination the Ginga and $A S C A$ data give reasonably tight constraints on the spectral slope of the hard continuum, $\alpha$, albeit in a model dependent way (as illustrated by the spread in values in Table 3). In practice we cannot exclude modest temporal variations in $\alpha$ on the basis of the available datasets.

The two "untied" parameters in the spectral fitting were the normalisation of the intrinsic power-law continuum and the flux of the iron $\mathrm{K}_{\alpha}$ line. The inferred absorption corrected flux of the hard X-ray source in the 2$10 \mathrm{keV}$ band at the time of the Ginga observations is 6.1 $\times 10^{-11}$ erg $\mathrm{cm}^{-2} \mathrm{~s}^{-1}$, whereas in the ASCA observations the value is $1.3 \times 10^{-11} \mathrm{erg} \mathrm{cm}^{-2} \mathrm{~s}^{-1}$ (these and subsequent values quoted in this section are based on Model 5). Thus variability in the continuum level by a factor $\sim 4.7$ is implied. As previously noted by Iwasawa et al. (1994), an observation by BBXRT (Marshall et al. 1991) made roughly 14 months after the Ginga observation gave an X-ray flux a factor 2 below the Ginga level.

The observed iron-line flux decreased by a factor of 1.8 between the Ginga and ASCA observations, substantially less than the corresponding continuum variation. Consequently the observed equivalent width of the iron $\mathrm{K}_{\alpha}$ line (measured relative to the absorbed continuum) shows an increase from 425 to $1110 \mathrm{eV}$ over the same interval. The measured iron-line energy, corrected for a redshift $z=0.0137$, is 
$E_{K \alpha}=6.38 \pm 0.03 \mathrm{keV}$ consistent with neutral iron. A possible origin for the iron $\mathrm{K}_{\alpha}$ emission is in the fluorescence of an extensive distribution of cold gas surrounding the nucleus of Mrk3, possible in the form of a torus. Our specific line of sight to the the source of the hard $\mathrm{X}$-ray continuum gives a column density $N_{H}=7.7 \times 10^{23} \mathrm{~cm}^{-2}$, which if applicable over $4 \pi$ steradians implies an iron-line equivalent width of $\sim 500 \mathrm{eV}$ (Leahy \& Creighton 1993). Given the uncertainty as to whether the Ginga or ASCA continuum level is more representative of the average long-term luminosity of Mrk 3 and our lack of detailed knowledge concerning both the coverage fraction and iron abundance of the fluorescing gas, it is plausible that the bulk of the observed iron line emission originates in this fashion. However, Compton reflection from the surfaces of optically thick media, such as a putative accretion disk or the inner walls of sections of the torus, may also contribute to the $6.4 \mathrm{keV}$ iron line. Our comparison of the Ginga and ASCA data demonstrates that we see hard X-ray variability down to $\sim 4.5 \mathrm{keV}$ (see Fig. 2) in Mrk 3 and suggests that we have a direct view of the power-law continuum (albeit with some losses due to the line-of-sight absorption) down to this energy. In this case the predicted contribution to the equivalent width of the iron line measured with respect to the observed continuum is $\lesssim 300 \mathrm{eV}$ (e.g. George \& Fabian 1991; Krolik \& Życki 1994; Ghisellini, Haardt \& Matt 1994). The observed decline of the iron line flux between the Ginga and ASCA observations demonstrates that this line does show some response on timescales of a few years, which in turn requires at least one of the line emitting regions to have a dimension not much more than about a light year. It would seem more likely that this scale size corresponds to an accretion disk or inner (optically thick) cloud structure rather than the inner extent of the putative molecular torus. In any event, milliarcsec resolution will be required to image this spatial structure.

The profile of the iron $\mathrm{K}_{\alpha}$ line in Mrk 3 is probably complex. For example, in the archetypal Seyfert 2 galaxy, NGC 1068, up to four line components have been spectrally resolved in the 6-7 keV energy band in ASCA observations (with a summed line photon flux which is $\sim 3$ times higher than is the case for Mrk 3) (Ueno et al. 1994; Iwasawa, Fabian \& Matt 1997). The identified line features include a $6.4 \mathrm{keV}$ line, probable He-like and $\mathrm{H}$-like iron $\mathrm{K}_{\alpha}$ components and also the hint of a "Compton shoulder" feature on the $6.4 \mathrm{keV}$ line (Iwasawa, Fabian \& Matt 1997). The He-like and H-like lines have been attributed to a warm scattering region (Marshall et al. 1993; Matt, Brandt \& Fabian 1996) which is probably smaller in extent than the optical/UV mirror in this source (Iwasawa, Fabian \& Matt 1997). Some evidence for underlying complexity in the iron $\mathrm{K}_{\alpha}$ line is provided, in the case of Mrk 3, by the fact that in the spectral fitting we minimize $\chi^{2}$ when we fix the width of the $6.4 \mathrm{keV}$ at a measureable value, namely $\sigma_{K \alpha}=0.1 \mathrm{keV}$. (In Model 5 , Table 3 the $\chi^{2}$ increased by $\sim 6$ when we reduced the line-width by a factor of 2 ). The origin of such broadening could be due to relativistic effects in an accretion disk (e.g. Tanaka et al. 1995; Yaqoob et al. 1995; Nandra et al. 1997) or the superposition of different line components. For example, our photoionized scatterer model predicts up to $20 \%$ of the iron $\mathrm{K}_{\alpha}$ flux observed by $A S C A$ will be in the form of
Fe XVIII - XXV, although this does not account for all of the apparent line broadening in Mrk 3.

Although previous studies have suggested that the power-law continuum in Mrk 3 is unusually hard (e.g. Awaki et al. 1990; Awaki et al. 1991; Smith \& Done 1996), as discussed in section 3.3, we obtain a value for the energy spectral index, $\alpha \sim 0.8$, which is well within the range of values seen in Seyfert 1 galaxies (Nandra \& Pounds 1994; Reynolds 1997; George et al. 1998). Even without reflection we require $\alpha \sim 0.7$ if we include an inner warm-absorber so as to reproduce the $\sim 8 \mathrm{keV}$ absorption edge apparent in the Ginga spectra. Our best fitting reflection model (Model 6, Table 3) requires a reflection component of only modest strength (i.e. $R \sim 0.8$ ), which contributes significantly to the observed spectrum only above $10 \mathrm{keV}$. In a recent study Turner et al. (1997b) suggest an alternative description of the hard X-ray spectrum of Mrk 3 in which the reflection component is effectively free of absorption and contributes the bulk of the observed flux between $3-10 \mathrm{keV}$. In this model the intrinsic power-law continuum suffers line-of-sight absorption by a cold gas column density $N_{H} \approx 1.3 \times 10^{24} \mathrm{~cm}^{-2}$ (roughly a factor of 2 higher than reported here) and is thus strongly suppressed below $\sim 6 \mathrm{keV}$. The current analysis demonstrates that continuum variability is observed in Mrk 3 down to $\sim 4.5 \mathrm{keV}$ with little evidence for a changing spectral form. If the continuum above $4.5 \mathrm{keV}$ represents the combination of reflection and a very heavily obscured power-law component, as in the Turner et al model, this would require the reflection component to track the power-law at a rate at least comparable to the intrinsic variability timescale (i.e. a timescale shorter than $\sim$ a year). One advantage of the Turner et al. (1997a) model is that the large iron-line equivalent width measured by $A S C A$ can be readily explained; however, as discussed earlier, when the continuum variability is taken into account, the ASCA measurement can also be reconciled with the current model in which the power-law component is observed directly down to $\sim 4.5 \mathrm{keV}$.

\subsection{The nature of the soft X-ray emission in Mrk 3}

The soft X-ray excess in the spectrum of Mrk 3 (which dominates the spectrum below $\sim 3 \mathrm{keV}$ ) has been modelled either as a blend of an electron scattered power-law continuum with a soft thermal component (Model 4) or an absorbed continuum scattered from a hot photoionized medium, with line emission from the same region superimposed (Model 5). We now consider each of these scenarios in turn.

In Model 4, a substantial fraction of the soft X-ray luminosity is explained as emission from a Raymond-Smith plasma at a temperature of $\sim 0.7 \mathrm{keV}$. A possible origin of this emission is in shock heated gas associated with a nuclear starburst in Mrk 3. The ROSAT HRI measurements of Morse et al. (1995) constrain the X-ray emitting region in Mrk 3 to be $<4 \mathrm{kpc}$, and thus do not exclude the presence of a nuclear starburst. In a recent study of the $A S C A$ spectra of a large sample of Seyfert 2/Narrow Emission Line Galaxies, Turner at al. (1997a) used far infra-red luminosities to estimate an upper limit to the fraction of the soft X-ray flux that could arise from starburst activity (following the method first discussed by David, Jones \& Forman 1992). On this basis Turner et al. (1997a) concluded that in 
Mrk 3 no more than $6 \times 10^{40} \mathrm{erg} \mathrm{s}^{-1}$ in the $0.5-4.5 \mathrm{keV}$ band could be thermal emission powered by starbursts, which represents only $5 \%$ of the total $0.5-4.5 \mathrm{keV}$ luminosity of the source. The thermal component in Model 4 has a $0.5-4.5$ $\mathrm{keV}$ luminosity of $2.1 \times 10^{41} \mathrm{erg} \mathrm{s}^{-1}$ (corrected for Galactic absorption), a factor 3.5 higher than the prediction. However the scatter in the correlation between the soft X-ray and farinfrared luminosity in the sample of normal and starburst galaxies studied by David et al. (1992) is of the same order; hence we conclude that a starburst origin for the thermal emission is not excluded. An alternative possibility is that the thermal emission arises in gas which is energised by the radio jet in Mrk 3 and possibly associated with the smallscale bar $\left(0.35^{\prime \prime} \times 1^{\prime \prime}\right)$ of continuum emission seen in the recent HST observation of Mrk 3 (Capetti et al. 1995).

The Raymond-Smith component contributes $23 \%$ of the observed $0.3-3 \mathrm{keV}$ emission (see Fig. 3(a)) with the electron scattered component accounting for the remainder. The scattered fraction, $f_{\text {scat }}$, of the hard continuum flux is $A_{\text {soft }} / A_{\text {Ginga }} \approx 2.7 \%$ (or as high as $\approx 12 \%$ if the level of the hard continuum in the ASCA observation is more representative of the time averaged behaviour of the source). For comparison Schmidt and Miller (1985) estimate that the scattered fraction is $<10 \%$ based on the degree of polarization in the optical spectrum of Mrk 3. The column density of the scattering medium can be estimated from the fraction $f_{\text {scat }}$, since $f_{\text {scat }}=\frac{\Omega}{4 \pi} \tau_{s}$ where $\Omega$ is the solid angle subtended by the scatterer (as viewed from the continuum source) and $\tau_{s}$ is the electron scattering optical depth given by $\tau_{s}=1.2 \sigma_{T} N_{\text {Hscat }}$. If we use the lower of the two estimates for the scattered fraction and take $\frac{\Omega}{4 \pi}=0.25$ then we obtain $N_{\text {Hscat }} \sim 1.3 \times 10^{23} \mathrm{~cm}^{-2}$.

We now turn to our alternative description of the soft $\mathrm{X}$-ray spectrum of Mrk 3. In Model 5 the scattering region is not completely photoionized and thus emprints both absorption and emission features on the emergent spectrum. The value for the column density, $N_{H_{\text {scat }}} \sim 1.7 \times 10^{23} \mathrm{~cm}^{-2}$, obtained from the spectral fitting is consistent with the previous estimate of a scattered fraction of $\sim 3 \%$. The ionization parameter of $\log \xi \sim 2.8$ corresponds to iron ionization states in the range Fe XVIII - XXV and oxygen either in a hydrogenic or fully ionized form (O VIII - IX) (Kallman \& McCray 1982). Although this is on the high side of the range, it is not totally atypical of the degree of ionization inferred for the warm absorbers seen in Seyfert 1 galaxies (Reynolds 1997; George et al. 1998). We have assumed that the plasma temperature is $10^{6} \mathrm{~K}$, which is hotter than the warm scattering which acts as an optical/UV mirror in NGC 1068 (Miller, Goodrich \& Mathews 1991). This is also above the limit of thermally stability in the photoionization models considered by Netzer $(1993,1996)$. However, the plasma state is similar to the case considered by Krolik \& Kriss (1995) (their model a), where the scattering region is identified as an Xray heated wind which is in ionization equilibrium but not necessarily radiative equilibrium. Empirically we find that reducing the plasma temperature to either $10^{5.5}$ or $10^{5} \mathrm{~K}$ produces a set of photoionization models which provide a somewhat poorer best fit to the observational data, particularly near the $0.9 \mathrm{keV}$ feature (see below), although many of the details of the spectral model are similar for plasma temperatures in the range $10^{5-6} \mathrm{~K}$. Tran (1995) notes that the observed FWHM of the polarized broad $\mathrm{H} \beta$ in Mrk 3 is $6000 \mathrm{~km} \mathrm{~s}^{-1}$, implying that the temperature of the electrons which scatter the optical light is less than $4 \times 10^{5}$ $\mathrm{K}$. The confirmation of a $10^{6} \mathrm{~K}$ component through X-ray spectroscopy might thus require thermal stratification of the scattering medium.

The observed feature in the $A S C A$ spectrum near $\sim 0.9$ $\mathrm{keV}$ is explained as recombination directly into the $\mathrm{K}$-shell of fully ionized oxygen atoms; the resulting O VIII recombination continuum is broadened by the thermal energies of the electrons in the $10^{6} \mathrm{~K}$ plasma. Fig. 3(b) (and also Fig $3(\mathrm{a})$ ) also shows a prominent line feature at $0.65 \mathrm{keV}$ due to the $\mathrm{O}$ VIII $\mathrm{Ly}_{\alpha}$ line, with an equivalent width of $\sim 30$ $\mathrm{eV}$. The observational evidence for this feature is marginal given that when we remove the line from the spectral model the result is a change in the $\chi^{2}$ of less than 1 . Similarly the model also predicts weak spectral features in the energy band from $1.8-3 \mathrm{keV}$ due to silicon and sulphur which are currently at the limit of detectability with ASCA.

In a recent paper, Comastri et al. (1997) considered the $A S C A$ spectrum of the Seyfert 2 galaxy NGC 4507 in which a line-like feature is again observed near $0.9 \mathrm{keV}$. These authors attribute this feature in NGC 4507 to a helium-like Neon line at a rest energy of $0.92 \mathrm{keV}$. In this case OVIII recombination at $0.87 \mathrm{keV}$ is formally inconsistent (at the $3 \sigma$ level) with the observed line energy. However, Comastri et al. (1997) note that in a $10^{6} \mathrm{~K}$ plasma, the broadening of the OVIII feature would have the effect of shifting the centroid energy to higher energy and therefore could be made consistent with the NGC 4507 observations. In fact we find, from a preliminary analysis of the relevant datasets, that the composite Ginga, ASCA and ROSAT spectrum of NGC 4507 can be well described by Model 5, with parameter values quite similar to those pertaining in Mrk 3.

The scale of the X-ray scattering region in Mrk 3 can be estimated from the derived value of the ionization parameter and the model assumptions. We obtain a radius $r=5 \times 10^{17} n_{6}^{-0.5} \mathrm{~cm}$ (where $n_{6}$ is the density in units of $n=10^{6}$ hydrogen nuclei $\mathrm{cm}^{-3}$ ). The measured column density through the scattering region leads to an estimate of the thickness of the scatterer of $l=1.7 \times 10^{17} n_{6}^{-1.0} \mathrm{~cm}$. Then if we require $l \lesssim r$, it follows that $n_{6} \gtrsim 0.1$. The implied scale of the scattering region $(\sim$ a light year $)$ is comparable to the inner extent of the cold fluorescing gas as inferred from the variability in the iron-line flux.

Finally we note the complication that the predicted normalisation of the emission spectrum is a factor $\sim 2$ times higher than the best fitting value. This apparent preference for scattered continuum as opposed to intrinsic emission from the photoionized region may reflect the fact that our representation of the spectral form of the incident continuum in the soft X-ray band, as a straight downward extrapolation of the hard power-law, may not be adequate. For example, the (hidden) intrinsic nuclear spectrum in Mrk 3 may show an upturn somewhere in the $0.3-3 \mathrm{keV}$ band similar to that seen in some unobscured Seyfert 1 nuclei (e.g. George et al. 1998).

\section{CONCLUSIONS}

The main results of our analysis of the composite Ginga, $A S C A$ and ROSAT spectrum of Mrk 3 are as follows: 
R. G. Griffiths et al.

(i) The broad-band $0.1-30 \mathrm{keV} \mathrm{X}$-ray spectrum is rather stable with only the level of the hard X-ray continuum and the iron $\mathrm{K}_{\alpha}$ line-flux showing clear evidence of variability on a timescale of years.

(ii) The iron $K_{\alpha}$ line energy is consistent with neutral iron. A component of this line emission may originate in the fluorescence of an extensive cold gas distribution, which has a column density $N_{H} \sim 7 \times 10^{23} \mathrm{~cm}^{-2}$ along our line of sight to the active nucleus; it is plausible that this gas is associated with a molecular torus in Mrk 3. Photoionization of the inner reaches of this medium may give rise to the "warm" absorption edge observed near $8 \mathrm{keV}$. The observed changes in the line equivalent width demonstrate that the iron line has a delayed response with respect to the continuum variations but, nevertheless, that iron-line reprocessing, possibly in an accretion disk, occurs within $\sim$ a light-year of the active nucleus in Mrk 3.

(iii) The measured iron line width of $\sigma_{K \alpha} \approx 0.1 \mathrm{keV}$ provides a hint of underlying complexity in composition of the iron $\mathrm{K}_{\alpha}$ line, possibly similar to the situation in NGC 1068. (iv) The inferred slope of the intrinsic hard X-ray continuum is model dependent with either a modest reflection component or the inclusion of an inner warm-absorber component both serving to steepen the derived spectral index. The continuum appears not to be anomalously flat compared to the norm for Seyfert 1 galaxies.

(v) The soft X-ray spectrum below $\sim 3 \mathrm{keV}$ is quite complex and may represent a blend of two or more separate components. A possible scenario is that the emission arises from the scattering of the nuclear continuum by a hot photoionized plasma which emprints both absorption and emission features on the emergent spectrum. In this setting the spectral feature present in the ASCA spectrum near $0.9 \mathrm{keV}$ is identified with OVIII recombination.

Clearly there is much to be learnt about Mrk 3 and other Seyfert 2 galaxies from X-ray spectral observations affording an appropriate combination of high spectral resolution, high sensitivity and broad-bandwidth coverage. Fortunately future missions such as AXAF, XMM and ASTRO-E will provide the opportunity to fully exploit this window on the Seyfert phenomenon.

\section{ACKNOWLEDGEMENTS}

The authors would like to thank Tim Kallman for his advice on using XSTAR for this analysis. RGG acknowledges support from PPARC in the form of a research studentship. CD acknowledges support from a PPARC advanced fellowship. The X-ray spectral data used in this work were obtained from the Leicester Data Archive Centre (LEDAS) and the HEASARC facility at Goddard Space Flight Centre, USA.

\section{REFERENCES}

Antonucci R.R.J., Miller J.S., 1985, ApJ, 297, 621

Antonucci R.R.J., 1993, ARA\&A, 31, 473

Awaki H., Koyama K., Kunieda H., Tawara Y., 1990, Nature, 346, 544

Awaki H., Koyama K., Inoue H., Halpern J.P., 1991, PASJ, 43, 195

Capetti A., Macchetto F., Axon D.J., Sparks W.B., Boksenberg A., 1995, ApJ, 448, 600

Cappi M., Matsuoka M., Brinkmann W., Prieto M.A., Palumbo G.G., 1996, ApJ, 456, 141

Comastri A., Vignali C., Cappi M., Matt G., Audano R., Awaki H., Ueno S., 1997, MNRAS, in press

David L.P., Jones C., Forman W., 1992, ApJ, 388, 92

Elvis M., Lockman F.J., Wilkes B.J., 1989, AJ, 97, 777

George I.M., Fabian A.C., 1991, MNRAS, 249, 352

George I.M., Turner T.J., Netzer N., Nandra K., Mushotzky R.F., Yaqoob T., 1998, ApJS, 114, 73

Ghisellini G., Haardt F., Matt G., 1994, MNRAS, 267, 743

Iwasawa K., Yaqoob T., Awaki H., Ogasaka Y., 1994, PASJ, 46, L167

Iwasawa K., Fabian A.C., Matt G., 1997, MNRAS, 289, 443

Kallman T.R., McCray R., 1982, ApJS, 50, 263

Kallman T.R., Krolik J.H., 1997, XSTAR: A Spectral Analysis Tool. Version 1.40 of the User's Guide

Krolik J.K., Madau P., Życki P.T., 1994, 420, L57

Krolik J.H., Kriss G.A., 1995, ApJ, 447, 512

Lampton M., Margon B., Bowyer S., 1976, ApJ, 208, 177

Leahy D.A., Creighton J., 1993, MNRAS, 263, 314

Makino F., 1987, ApL, 25, 223

Marshall F.E., Netzer H., Arnaud K.A., Boldt E.A., Holt S.S., Jahoda K.M., Kelley R., Mushotzky R.F., Petre R., Serlemitsos P.J., Smale A.P., Swank J.H., Szymkowiak A.E., Weaver K.A., 1993, ApJ, 405, 168

Marshall F.E., Boldt, E.A., Holt S.S., Jahoda K.M., Kelley R.L., Mushotzky R.F., Petre R., Serlemitsos P.J., Smale A.P., Swank J.H., Szymkowlak A.E., Weaver K., 1991, Proc. 28th Yamada Conference, Nagoya, Japan, April 8-12, 1991, "Frontiers of X-ray Astronomy", eds. Y. Tanaka \& K. Koyama (universal Academy Press), p.233

Matt G., Brandt W.N., Fabian A.C., 1996, MNRAS, 280, 823

Miller J.S., Goodrich R.W., Mathews W.G., 1991, ApJ, 378, 47

Morrison R., McCammon D., 1983, ApJ, 270, 119

Morse, J.A., Wilson, A.S., Elvis, M., Weaver K.A., 1995, ApJ, 439, 121

Nandra K., Pounds K.A., 1994, MNRAS, 268, 405

Nandra K., George I.M., Mushotzky R.F., Turner T.J., Yaqoob T., 1997, ApJ, 477, 602

Netzer H., 1993, ApJ, 411, 594

Netzer H., 1996, ApJ, 473, 781

Pounds K.A., Nandra K., Stewart G.C., George I.M., Fabian A.C., 1990, Nature, 344, 132

Reynolds C.S., 1997, MNRAS, 286, 513

Schmidt G.D., Miller J.S., 1985, ApJ, 290, 517

Smith D.A., Done C., 1996, MNRAS, 280, 355

Tanaka Y., Inoue H., Holt S.S., 1994, PASJ, 46, L37

Tanaka Y., Nandra K., Fabian A.C., Inoue H., Otani C., Dotani T., Hayashida K., Iwasawa K., Kii T., Kunieda H., Makino F., Matsuoka M., 1995, Nature, 375, 659

Tran, H.D., 1995, ApJ, 440, 597

Turner M.J.L., et al. 1989, PASJ, 41, 345

Turner T.J., Pounds K.A., 1989, MNRAS, 240833

Turner T.J., Urry C.M., Mushotzky R.F., 1993, ApJ, 418, 653

Turner T.J., George I.M., Nandra K., Mushotzky R.F., 1997a, ApJS, 113, 23

Turner T.J., George I.M., Nandra K., Mushotzky R.F., 1997b, ApJ, 488, 164

(C) 0000 RAS, MNRAS 000, 000-000 
Ueno S., Mushotzky R.F., Koyama K., Iwasawa K., Awaki H., Hayashi I., 1994, PASJ, 46, L71

Yaqoob T., Edelson R., Weaver K.A., Warwick R.S., Mushotzky R.F., Serlemitsos P.J., Holt S.S., 1995, ApJ, 453, L81

\section{Figure Captions}

Figure 1. Top panel: The Ginga LAC top-layer (triangles) and mid-layer (circles) count rate spectra for Mrk 3. The solid line corresponds to the best fitting version of Model 1. Bottom panel: The spectral fitting residuals to the Ginga data for Mrk 3 (scaled in terms of the measurement errors).

Figure 2. Top panel: The unfolded composite spectrum of Mrk 3 based on the best-fitting version of Model 3. Bottom panel: The spectral fitting residuals to the composite spectrum for Mrk 3. The data points correspond to Ginga top-layer (circles), Ginga mid-layer (squares), ASCA SIS-0 (crosses) and ROSAT PSPC (triangles). For clarity only the $A S C A$ SIS-0 points are shown.

Figure 3. Modelling of the soft X-ray (0.3-3 keV) spectrum of Mrk 3. (a) As a blend of a soft (scattered) power-law continuum with $\mathrm{a} \sim 0.7 \mathrm{keV}$ Raymond-Smith component. The spectrum from 0.7-0.9 keV is dominated by O, Fe (L shell) and Ne lines. (b) As an absorbed continuum scattered from a hot photoionized medium plus line emission from the same region. The feature near $0.9 \mathrm{keV}$ is due to O VIII recombination and that at $0.65 \mathrm{keV}$ corresponds to the $\mathrm{O}$ VIII $\mathrm{L}_{\alpha}$ line. 
Table 1. Spectral fitting of the Ginga data alone

Parameter Model $1 \quad$ Model 2

$\begin{array}{lcc}\alpha & 0.37_{-0.14}^{+0.15} & 0.65_{-0.19}^{+0.20} \\ A_{\text {Ginga }}{ }^{a} & 0.6_{-0.2}^{+0.3} & 1.5_{-0.6}^{+1.1} \\ N_{H}{ }^{b} & 58 \pm 7 & 69 \pm 9 \\ E_{\mathrm{K}_{\alpha}{ }^{c}{ }^{c}} & 6.39 \pm 0.10 & 6.33_{-0.21}^{+0.19} \\ A_{\mathrm{K}_{\alpha}}{ }^{-0.2} & 7.9 \pm 1.4 & 4.0_{-2.4}^{+2.2} \\ E_{\text {edge }}{ }^{c} & & \\ \tau_{\text {edge }} & - & 8.1_{-0.5}^{+0.4} \\ \chi^{2} & - & 0.24 \pm 0.10 \\ \text { d.o.f. } & 56.0 & 42.1 \\ & 56 & 54\end{array}$

a $10^{-2}$ photon $\mathrm{cm}^{-2} \mathrm{~s}^{-1} \mathrm{keV}^{-1}$.

b $10^{22} \mathrm{~cm}^{-2}$.

${ }^{c} \mathrm{keV}$.

${ }^{d} 10^{-5}$ photon $\mathrm{cm}^{-2} \mathrm{~s}^{-1}$.

Table 2. Spectral fitting of the ASCA SIS/GIS data alone

\begin{tabular}{|c|c|c|c|}
\hline Parameter & Model 3 & Model 4 & Model 5 \\
\hline $\begin{array}{l}\alpha \\
A_{A S C A}{ }^{a} \\
N_{H_{1}}{ }^{b}\end{array}$ & $\begin{array}{c}1.12 \pm 0.10 \\
0.45_{-0.09}^{+0.12} \\
41.4_{-5.3}^{+6.0}\end{array}$ & $\begin{array}{c}0.56 \pm 0.16 \\
0.14_{-0.05}^{+0.06} \\
44.0_{-6.8}^{+8.2}\end{array}$ & $\begin{array}{c}0.91_{-0.12}^{+0.11} \\
0.32_{-0.11}^{+0.11} \\
48.0_{-7.1}^{+8.7}\end{array}$ \\
\hline $\begin{array}{ll}E_{K_{\alpha}}{ }^{c} \\
A_{K_{\alpha}}{ }^{d}\end{array}$ & $\begin{array}{c}6.31 \pm 0.03 \\
4.2 \pm 0.6\end{array}$ & $\begin{array}{c}6.30 \pm 0.03 \\
4.2 \pm 0.6\end{array}$ & $\begin{array}{c}6.30 \pm 0.03 \\
4.1 \pm 0.6\end{array}$ \\
\hline$A_{\text {soft }}{ }^{a}$ & $0.035 \pm 0.002$ & $0.022 \pm 0.003$ & $0.037_{-0.005}^{+0.006}$ \\
\hline $\begin{array}{l}k T_{\text {Ray }}{ }^{c} \\
A_{\text {Ray }}{ }^{a}\end{array}$ & $\begin{array}{l}- \\
-\end{array}$ & $\begin{array}{c}0.74 \pm 0.06 \\
0.008_{-0.002}^{+0.002}\end{array}$ & - \\
\hline $\begin{array}{l}\log \xi \\
N_{H_{\text {scat }}} b \\
A_{\text {em }}{ }^{e}\end{array}$ & $\begin{array}{l}- \\
- \\
-\end{array}$ & $\begin{array}{l}- \\
- \\
-\end{array}$ & $\begin{array}{c}2.42_{-0.24}^{+0.27} \\
6_{-3}^{+5} \\
1.1 \pm 0.4\end{array}$ \\
\hline $\begin{array}{l}\chi^{2} \\
\text { d.o.f. } \\
10^{-2} \text { photo } \\
10^{22} \mathrm{~cm}^{-2} . \\
\mathrm{keV} . \\
10^{-5} \text { photo } \\
10^{-12} \text { phot }\end{array}$ & $\begin{array}{c}390.0 \\
300 \\
\mathrm{~cm}^{-2} \mathrm{~s}^{-1} \mathrm{ke}\end{array}$ & $\begin{array}{c}321.1 \\
298\end{array}$ & $\begin{array}{c}318.8 \\
297\end{array}$ \\
\hline
\end{tabular}


Table 3. Spectral fitting of the composite Ginga, ASCA and ROSAT spectra for Mrk 3

\begin{tabular}{|c|c|c|c|c|c|}
\hline Parameter & Model 3 & Model 4 & Model 5 & Model 6 & Model 7 \\
\hline $\begin{array}{l}\alpha \\
A_{\text {Ginga }}{ }^{a} \\
A_{A S C A}{ }^{a} \\
N_{H}{ }^{b}\end{array}$ & $\begin{array}{c}0.88 \pm 0.06 \\
2.58_{-0.40}^{+0.47} \\
0.64_{-0.12}^{+0.14} \\
83.4_{-3.5}^{+3.6}\end{array}$ & $\begin{array}{c}0.45 \pm 0.08 \\
0.77_{-0.16}^{+0.20} \\
0.17_{-0.04}^{+0.06} \\
66.4 \pm 4.1\end{array}$ & $\begin{array}{c}0.66 \pm 0.07 \\
1.39_{-0.25}^{+0.29} \\
0.29_{-0.07}^{+0.08} \\
77.0 \pm 4.0\end{array}$ & $\begin{array}{c}0.80_{-0.11}^{+0.07} \\
1.62_{-0.33}^{+0.29} \\
0.36_{-0.09}^{+0.08} \\
71.4_{-4.8}^{+4.6}\end{array}$ & $\begin{array}{c}0.69 \pm 0.07 \\
1.63_{-0.28}^{+0.26} \\
0.34_{-0.07}^{+0.08} \\
66.8_{-3.7}^{+6.6}\end{array}$ \\
\hline $\begin{array}{l}E_{K_{\alpha}}{ }^{c} \\
A_{K \alpha}(\text { Ginga })^{d} \\
A_{K \alpha}(\text { ASCA })^{d}\end{array}$ & $\begin{array}{c}6.29 \pm 0.03 \\
6.0_{-1.5}^{+1.4} \\
3.8 \pm 0.6\end{array}$ & $\begin{array}{c}6.30 \pm 0.03 \\
7.6 \pm 1.4 \\
4.1 \pm 0.6\end{array}$ & $\begin{array}{c}6.29 \pm 0.03 \\
7.0 \pm 1.4 \\
3.9 \pm 0.6\end{array}$ & $\begin{array}{c}6.29 \pm 0.03 \\
5.7 \pm 1.5 \\
3.8_{-0.5}^{+0.6}\end{array}$ & $\begin{array}{c}6.28 \pm 0.03 \\
5.2 \pm 1.5 \\
3.8 \pm 0.6\end{array}$ \\
\hline$A_{\text {soft }}{ }^{a}$ & $0.033 \pm 0.001$ & $0.021 \pm 0.002$ & $0.038 \pm 0.004$ & $0.039 \pm 0.004$ & $0.039_{-0.004}^{+0.005}$ \\
\hline $\begin{array}{l}k T_{\text {Ray }}{ }^{c} \\
A_{\text {Ray }}{ }^{a}\end{array}$ & $\begin{array}{l}- \\
-\end{array}$ & $\begin{array}{c}0.69_{-0.05}^{+0.07} \\
0.0085_{-0.0011}^{+0.0018}\end{array}$ & $\begin{array}{l}- \\
-\end{array}$ & - & - \\
\hline $\begin{array}{l}\log \xi \\
N_{H_{\text {scat }}} b \\
A_{\text {em }}^{e}\end{array}$ & $\begin{array}{l}- \\
- \\
-\end{array}$ & $\begin{array}{l}- \\
- \\
-\end{array}$ & $\begin{array}{c}2.81 \pm 0.13 \\
17_{-5}^{+6} \\
0.9 \pm 0.2\end{array}$ & $\begin{array}{c}2.61_{-0.08}^{+0.23} \\
10_{-3}^{+7} \\
0.9 \pm 0.2\end{array}$ & $\begin{array}{c}2.78_{-0.19}^{+0.12} \\
16_{-6}^{+5} \\
0.9 \pm 0.2\end{array}$ \\
\hline$N_{H_{\text {warm }}}^{b}$ & - & - & - & - & $39_{-18}^{+1^{*}}$ \\
\hline$R$ & - & - & - & $0.8 \pm 0.5$ & - \\
\hline $\begin{array}{l}\chi^{2} \\
\text { d.o.f. }\end{array}$ & $\begin{array}{c}581.0 \\
378\end{array}$ & $\begin{array}{c}417.6 \\
376\end{array}$ & $\begin{array}{c}405.6 \\
375\end{array}$ & $\begin{array}{c}397.0 \\
374\end{array}$ & $\begin{array}{c}391.2 \\
374\end{array}$ \\
\hline
\end{tabular}

${ }^{a} 10^{-2}$ photon $\mathrm{cm}^{-2} \mathrm{~s}^{-1} \mathrm{keV}^{-1}$.

${ }^{b} 10^{22} \mathrm{~cm}^{-2}$.

${ }^{c} \mathrm{keV}$.

${ }^{d} 10^{-5}$ photon $\mathrm{cm}^{-2} \mathrm{~s}^{-1}$.

e $10^{-12}$ photon $\mathrm{cm}^{-2} \mathrm{~s}^{-1}$.

* reached upper limit of model. 


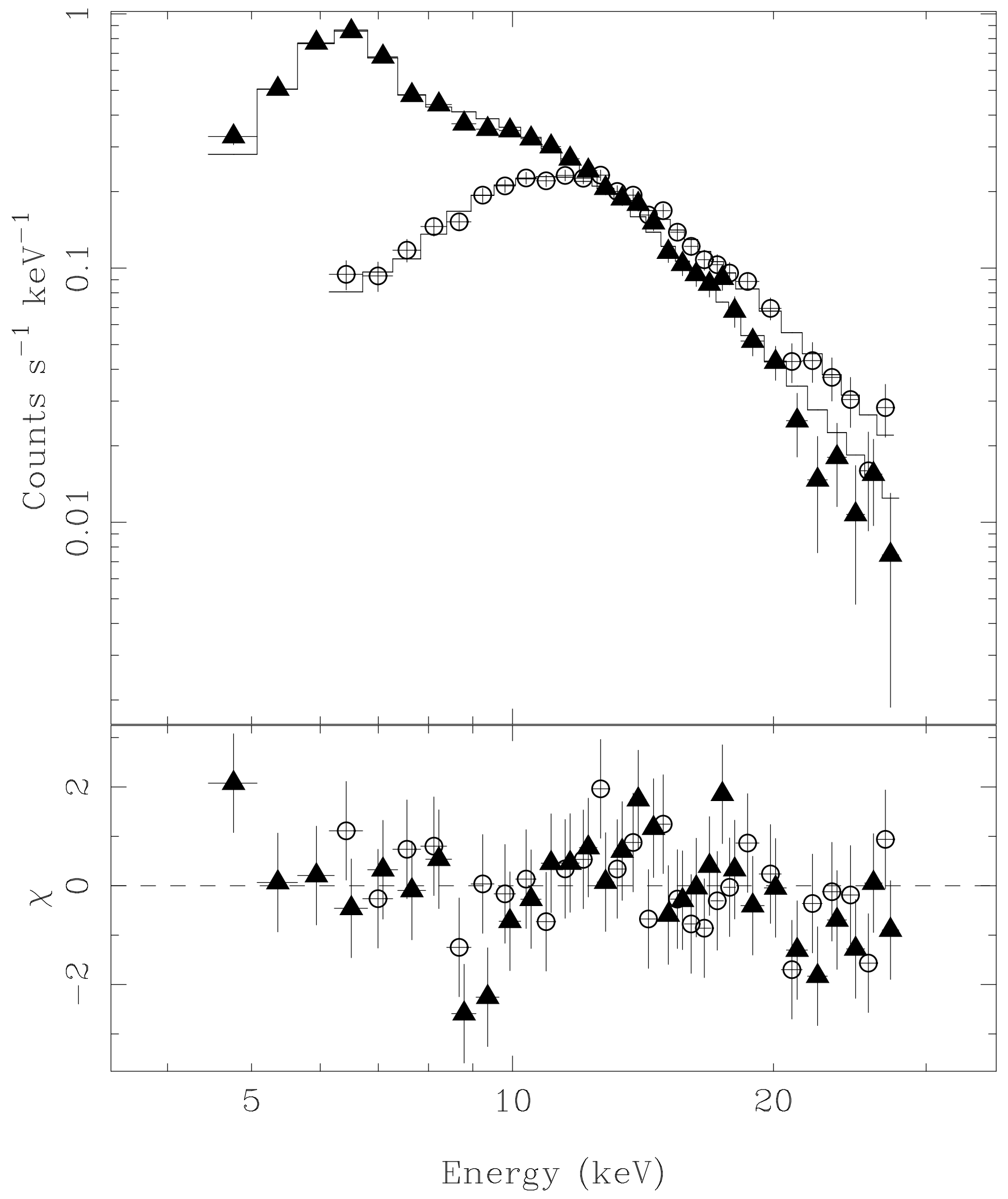




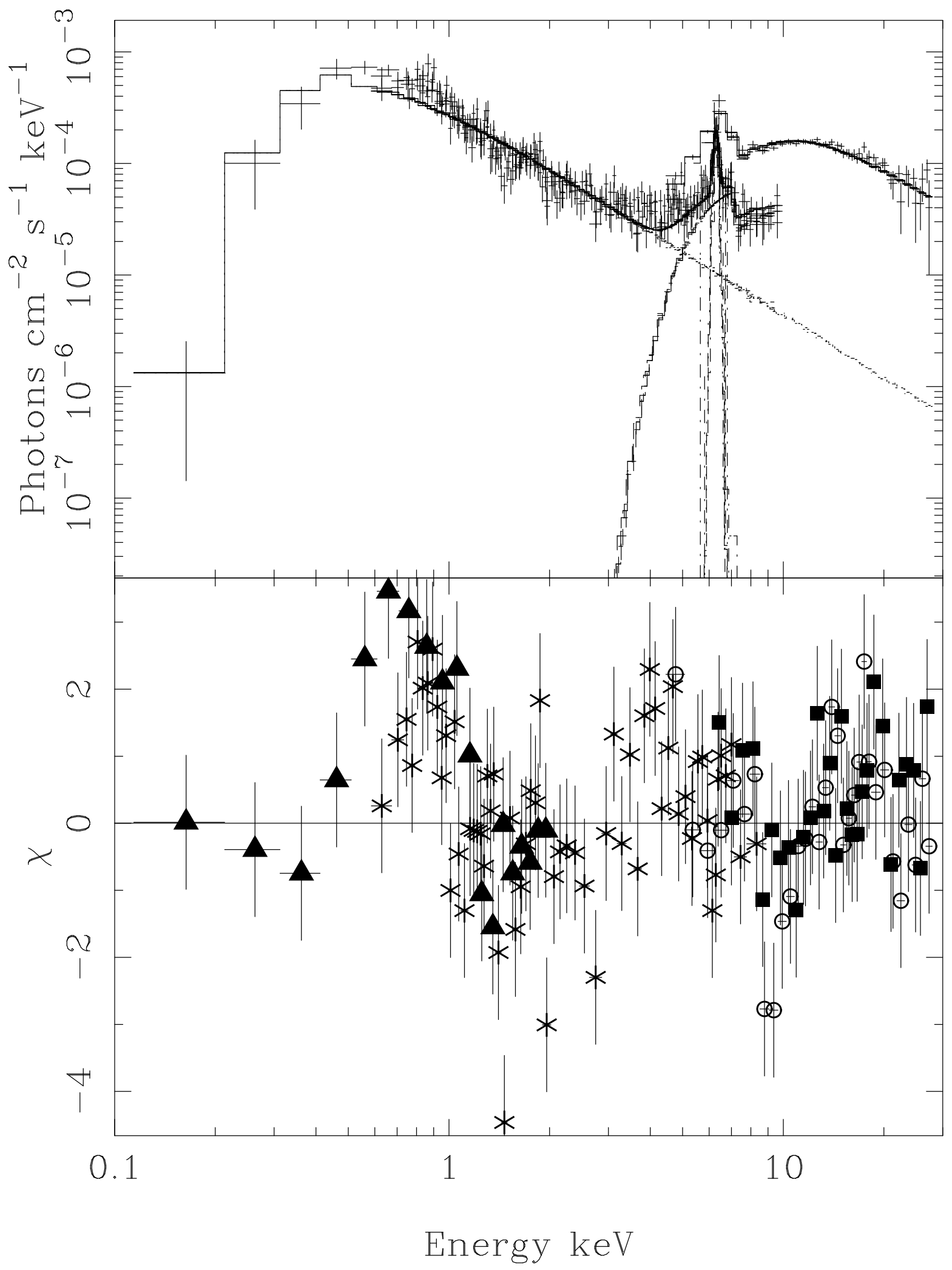




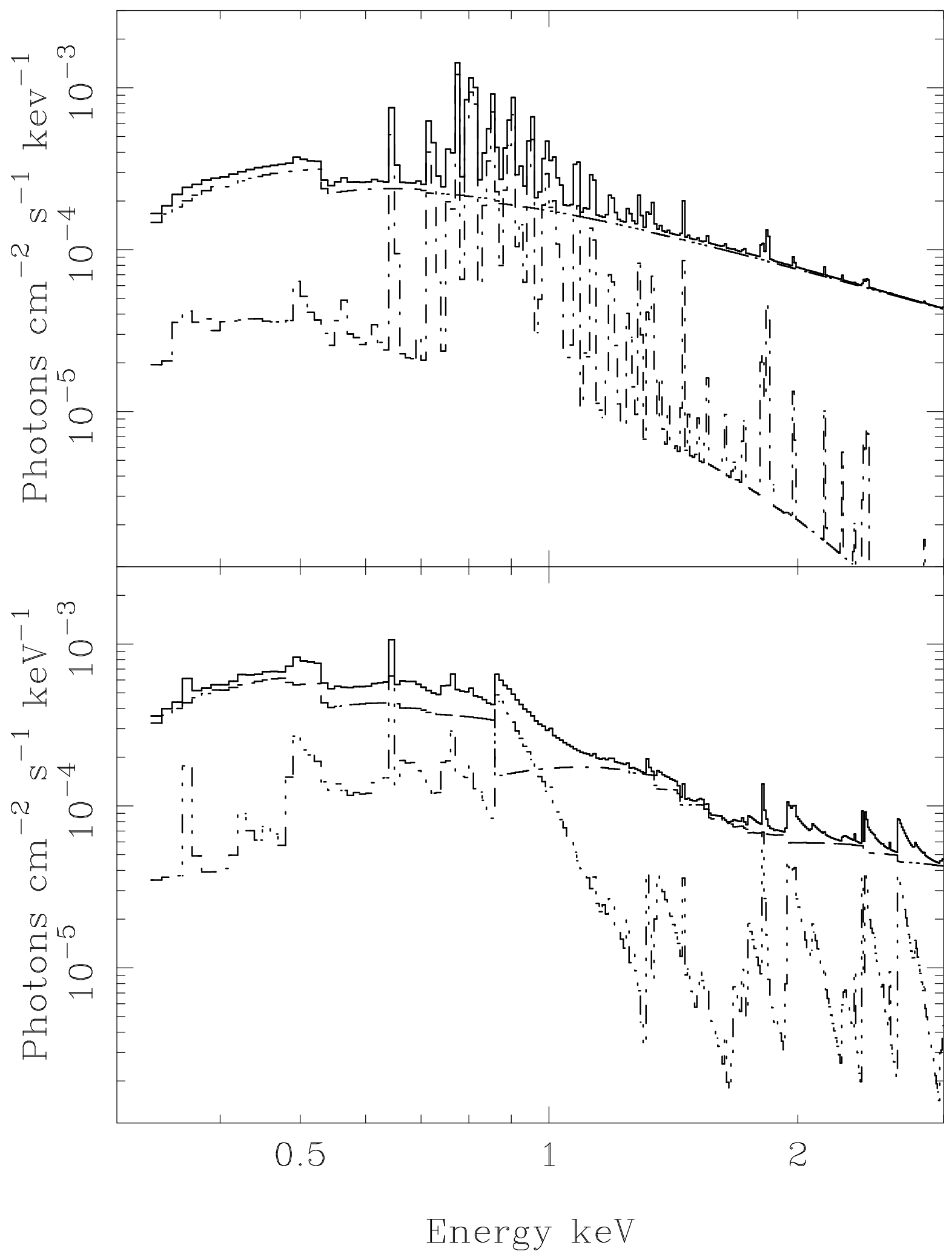

